\title{
Microbiota, a key player in alcoholic liver disease
}

\author{
Anne-Marie Cassard ${ }^{1,2}$ and Dragos Ciocan ${ }^{1,2}$ \\ ${ }^{1}$ INSERM UMR996, Inflammation, Chemokines, and Immunopathology, Clamart, France; ${ }^{2}$ Univ Paris-Sud, Univ Paris-Saclay, DHU \\ Hepatinov, Labex Lermit, CHU Bicêtre, Kremlin-Bicêtre, France
}

Alcoholic liver disease (ALD) is a major cause of morbidity and mortality worldwide. Only $20 \%$ of heavy alcohol consumers develop alcoholic liver cirrhosis. The intestinal microbiota (IM) has been recently identified as a key player in the severity of liver injury in ALD. Common features of ALD include a decrease of gut epithelial tight junction protein expression, mucin production, and antimicrobial peptide levels. This disruption of the gut barrier, which is a prerequisite for ALD, leads to the passage of bacterial products into the blood stream (endotoxemia). Moreover, metabolites produced by bacteria, such as short chain fatty acids, volatile organic compounds (VOS), and bile acids (BA), are involved in ALD pathology. Probiotic treatment, IM transplantation, or the consumption of dietary fiber, such as pectin, which all alter the ratio of bacterial species, have been shown to improve liver injury in animal models of ALD and to be associated with an improvement in gut barrier function. Although the connections between the microbiota and the host in ALD are well established, the underlying mechanisms are still an active area of research. Targeting the microbiome through the use of prebiotic, probiotic, and postbiotic modalities could be an attractive new approach to manage ALD. (Clin Mol Hepatol 2018;24:100-107)

Keywords: Alcoholic liver disease; Intestinal microbiota; Probiotics; Pectin; Fiber

\section{INTRODUCTION}

Excessive alcohol consumption is a leading cause of chronic liver disease worldwide. Alcoholic liver disease (ALD) ranges from hepatic steatosis to steatohepatitis, fibrosis, cirrhosis, and, potentially, hepatocellular carcinoma (HCC). Fatty liver, known as steatosis, is due to the accumulation of triglycerides in the cytoplasm of hepatocytes and is reversible after withdrawal of alcohol intake. However, it may progress towards inflammation in chronic drinkers. ${ }^{1}$ The inflammatory process progresses towards fibrosis and cirrhosis in approximately $20 \%$ of alcoholic patients and the incidence of HCC in cirrhotic livers is approximately 3 to $5 \%$ each year. ${ }^{2}$ Some ALD patients develop severe alcoholic hepatitis (SAH), characterized by acute hepatocellular insufficiency, severe histological liver injury, and a high mortality rate, as the one-month survival of such patients is between 50 and $65 \%$.

Treatment of ALD relies principally on alcohol withdrawal and there is no treatment that can prevent lesion progression towards cirrhosis in active drinkers. Patients with SAH are treated with corticosteroids, although the most recent data showed no improvement in mortality at 90 days or one year. ${ }^{3}$ The use of pentoxifylline, a phosphodiesterase inhibitor or $\mathrm{N}$-acetylcysteine

\footnotetext{
Abbreviations:

ALD, alcoholic liver disease; AH, alcoholic hepatitis, ASCA, anti-Saccharomyces cerevisiae antibodies; BA, bile acids; CDR, cirrhosis dysbiosis ratio; CDCA, chenodeoxycholic acid; FMT, fecal microbiota transfer; FXR, farnesoid X receptor; HCC, hepatocellular carcinoma; HE, hepatic encephalopathy; IM, intestinal microbiota; LCFA, long-chain fatty acids; LPS, Lipopolysaccharides; Reg3 $\beta$, regenerating islet-derived protein 3 beta; Reg3 $\gamma$, regenerating islet-derived protein 3 gamma; $\mathrm{SAH}$, alcoholic patient with severe alcoholic hepatitis; SCFA, short chain fatty acids; TGR5, Takeda G protein-coupled receptor 5; TLR, Toll-like receptor; VOC, volatile organic compounds
}

\section{Corresponding author : Anne-Marie Cassard}

INSERM U996 Inflammation, Chemokines and Immunopathology, DHU Hepatinov, Univ Paris-Sud, Université Paris-Saclay, 32 rue des Carnets, 92140 Clamart, France

Tel: +33141288037, Fax: +33146327993

E-mail: cassard.doulcier@u-psud.fr.

http://orcid.org/0000-0002-1813-5128 
Anne-Marie Cassard, et al. Microbiota in alcoholic liver disease

(NAC), a glutathione precursor, alone or in association with corticosteroids, is controversial, as they don't improve survival. Enteral or parenteral nutritional supplementation may improve the prognosis, as ALD is often associated with protein, vitamin, and mineral deficiencies, but its impact on survival is still unclear. Liver transplantation can be considered in selected patients with a first episode of sAH, despite alcohol withdrawal, who do not respond to corticosteroids, but only after a thorough addictological assessment. ${ }^{4}$ It can also be an option in patients with end-stage disease or complications of alcoholic cirrhosis, but generally only after six months of abstinence. Its use is limited, however, due to the shortage of organs.

It has been suggested that factors other than the quantity of alcohol consumed may play a role in ALD, as not all chronic alcohol consumers develop liver injury. Genome-wide association studies have identified several risk loci in Patatin-like phospholipase domain-containing protein 3 (PNPLA3), transmembrane 6 superfamily 2 human gene (TM6SF2), membrane-bound 0-acyltransferase domain-containing protein 7 (MBOAT7), and sodium-coupled neutral amino acid transporter 4 (SLC38A4) genes that were associated with alcohol-related cirrhosis and SAH. ${ }^{5,6}$ However, the heritability of alcohol-related cirrhosis risk appears to be modulated through polygenic and complex inheritance in the presence of several environmental risk factors ${ }^{7}$ and cannot completely explain the difference between patients that develop ALD and those that do not. It has more recently been shown that the intestinal microbiota (IM) also contributes to ALD and could be a new therapeutic target for the treatment and cure of ALD patients.

\section{THE IM, A NEW PLAYER IN ALD}

In 1995, Adachi Y, et al. reported that antibiotics protected rats against alcohol-induced liver injury by lowering Kupffer cell activation, without linking its use to gut bacteria. ${ }^{8} A L D$ is also associated with elevated plasma endotoxin levels in alcoholic patients and rodent models of alcohol consumption. ${ }^{9-11}$ Endotoxemia reflects the level of lipopolysaccharides (LPS), a component of the bacterial cell wall of gram-negative bacteria. LPS binds to toll-like receptor (TLR)4 and TLR4 deficient mice exhibit less liver injury after alcohol intake than wildtype mice, demonstrating the role of bacterial products. ${ }^{12}$ Furthermore, increased endotoxemia also correlates with increased intestinal permeability. ${ }^{13}$ This is due to decreased expression of several proteins in the tight junctions in ALD models. ${ }^{14-16}$ Moreover, ethanol consumption modulates mucin glycosylation, which modifies the protective mucus layer and, potentially, the adherent bacterial species. ${ }^{17}$ It was shown, using germ-free mice, that the alcohol-associated increase of intestinal permeability is not induced by alcohol, per se, but at least in part by acetaldehyde produced by bacterial ethanol metabolism. ${ }^{18}$ These data do not exclude the possible involvement of other bacterial metabolites in the disruption of the gut barrier. ${ }^{19}$ Thus, the IM participates in the disruption of the gut barrier, which may impair liver homeostasis by increasing the level of bacterial products or bacterial translocation into the blood and lymph nodes. Such translocation may be particularly deleterious in the case of dysbiosis. Indeed, dysbiosis has already been described in alcohol-fed rodents. ${ }^{20-22}$ Alcohol intake in rats was associated with a decrease in the abundance of lactic acid bacteria, especially those of the genera Lactobacillus, Pediococcus, Leuconostoc, and Lactococcus. ${ }^{22} \mathrm{An}$ increase in the abundance of Proteobacteria and Actinobacteria and a decrease in that of Bacteroidetes and Firmicutes have been reported in alcohol-fed mice. Moreover, these modifications were accompanied by a decrease in bacterial diversity. ${ }^{20}$ Dysbiosis of the colon IM in alcoholic patients has been associated with a decrease in the abundance of Bacteroidetes and an increase in that of Enterobacteriaceae and Proteobacteria. ${ }^{23}$ These studies show that dysbiosis is associated with alcohol-induced liver lesions, but a causal role for the IM was only been recently demonstrated.

Patients with SAH harbor an IM that is different from that of alcoholic patients without liver lesions (without AH). ${ }^{24}$ The propensity of alcohol-induced inflammation was shown to be transmissible from patients to mice via the transplantation of the IM. Germfree mice that received the IM of an alcoholic patient with SAH developed more severe liver lesions than mice receiving the IM of an alcoholic patient without SAH. They also developed more pronounced disruption of the intestinal barrier, associated with visceral inflammation. Efficient IM transplantation of a human stool sample from an alcoholic patient without SAH into conventional mice reverted the development of liver lesions in alcohol-fed mice that initially received the IM of an alcoholic patient with $\mathrm{sAH} .{ }^{24}$ Altogether, these findings show that individual susceptibility to ALD is dependent on the IM and provide strong evidence for a causal role of the IM in ALD.

The connections between the microbiota and their host in the development of ALD have been established, but the mechanisms which are involved in the deleterious role mediated by the IM are still unclear. ${ }^{25}$ However, metabolites produced by bacteria, such as short chain fatty acids (SCFA), and, more importantly, volatile or- 
ganic compounds (VOC) and bile acids (BA), appear to be key players in ALD.

Chen $\mathrm{P}$, et al. showed in an animal model of ALD that alcoholfed mice exhibited decreased expression of cecal microbiota bacterial genes involved in the biosynthesis of saturated fatty acids and decreased levels of saturated long-chain fatty acids (LCFAs). ${ }^{26}$ Furthermore, dietary supplementation with saturated LCFAs restored eubiosis and the intestinal gut barrier, leading to an improvement of the liver injury. The authors confirmed these results in alcohol abuse patients and positively correlated LCFA levels with those of Lactobacilli in fecal samples, as saturated LCFAs are metabolized by commensal Lactobacillus and promote their growth. The composition of dietary fat also has an impact on the IM and liver injury. A diet enriched in unsaturated fats was shown to exacerbate ALD and induce dysbiosis (reduction in the abundance of Bacteroidetes and increase in that of Proteobacteria and Actinobacteria) and metabolomic changes (decreased levels of several LCFAs, medium-chain fatty acids (MCFAs), and SCFAs) in mice. ${ }^{27}$ These results suggest that dietary lipids may also play an etiological role in ALD by altering IM composition and function.

Analysis of the fecal metabolome from alcohol-fed mice has also highlighted the involvement of BA in ALD. ${ }^{24}$ Mice transplanted with the IM of a patient with SAH developed more severe liver lesions than those transplanted with the IM of an alcoholic patient without liver disease (without AH). Comparison of the fecal metabolites allowed the identification of 13 biomarkers related to alcohol-induced liver toxicity. The most discriminating molecules were BA derivatives and hydroxygenated/oxygenated fatty acids. Higher concentrations of the primary bile acid, chenodeoxycholic acid (CDCA), were found in fecal samples of mice without liver lesions than those of mice with alcohol-induced liver lesions. The secondary bile acid, ursodesoxycholic acid (UDCA), was more abundant in the fecal metabolome of mice that received the MI of the alcoholic patient without liver disease than of those that received the MI of the patient with ALD. More than $95 \%$ of the BA secreted in bile are reabsorbed by the distal ileum and return to the liver, taking part in the entero-hepatic cycle. The main function of BA is to assist the absorption of dietary lipids and lipid-soluble nutrients. However, they are also signaling molecules that act through the activation of receptors, such as the farnesoid $X$ receptor (FXR) or G protein-coupled receptor (TGR5). Thus, they may modulate the inflammatory response, and lipid, glucose, energy, and drug metabolism, as well as their own biosynthesis by a negative feed-back loop following FXR activation. ${ }^{28}$ The primary bile acid, CDCA, is the most potent, whereas secondary BA acti- vate the FXR and TGR5 to a much lesser extent. The metabolism of the IM may thus modulate FXR and TGR5 activation and subsequently ALD susceptibility.

Alcohol consumption is also associated with a specific mixture of VOC in feces. ${ }^{29}$ Amongst these, the SCFAs propionate and isobutyrate, involved in intestinal epithelial cell homeostasis and gut barrier integrity, are less abundant in the feces of alcoholics than that of non-alcoholic healthy individuals.

Disruption of the gut barrier is a prerequisite for ALD, resulting in bacterial translocation. ${ }^{30}$ The integrity of the gut epithelium is dependent on the balance between the IM, mucosal immunity, and mucus and antimicrobial peptide production and their alteration is associated with ALD. Furthermore, the production of mucin by goblet cells, which decreases in mice with alcohol-induced liver lesions, depends on the $\mathrm{IM}^{24}$ Surprisingly, mice deficient for mucin production have been shown to be protected against alcoholinduced liver lesions. This deficiency was associated with a large increase in defensin production, as well as that of lectins regenerating islet-derived protein 3 beta (Reg3 $\beta$ ) and regenerating isletderived protein 3 gamma (Reg3 $\gamma$ ), which led to increased destruction of commensal bacteria and prevented bacterial overgrowth. ${ }^{31}$ Moreover, defensin, Reg3 $\beta$, and Reg3 $\gamma$ levels decreased in mice fed alcohol ${ }^{22}$ and mice deficient for Reg $3 \beta$ and Reg $3 \gamma$ showed an increased abundance of adherent bacteria and a high level of bacterial translocation, promoting alcohol-induced liver lesions. ${ }^{32}$ Conversely, mice that overexpressed Reg $3 \gamma$ were protected from alcohol-induced hepatic toxicity, associated with a decreased abundance of adherent bacteria and a low level of bacterial translocation. Moreover, mice harboring a specific IM that protected them from the hepatic toxicity of alcohol also expressed high levels of defensins. ${ }^{33}$ These data suggest that the control of bacterial overgrowth and the abundance of adherent bacteria are essential for the protective mechanisms against alcohol-induced liver toxicity.

Recent data have also emphasized the role played by the IM in sensing nutrients and hormones via the gastrointestinal nervous system. ${ }^{34-36}$ The increase in intestinal permeability and dysbiosis in alcoholic patients has been associated with higher scores of depression, anxiety, and alcohol craving. ${ }^{37}$ Alcoholic patients with elevated gut permeability had a lower abundance of genera belonging to the Ruminococcaceae family (Ruminococcus, Faecalibacterium, Subdoligranulum, Oscillibacter, and Anaerofilum), as well as Clostridia, than patients with less gut permeability. Conversely, the abundance of Dorea (Lachnospiraceae), Blautia, and Megasphaera was higher in these patients. Furthermore, the lev- 
els of VOCs (especially indole and phenol species) produced by these bacteria were altered in alcoholic patients. This suggests that metabolites produced by the IM are involved not only in cell dysfunction and liver disease, but also the psychological symptoms of alcohol dependence. ${ }^{37}$

\section{ALD AND FUNGAL DYSBIOSIS}

Recent studies have also shown the importance of changes in the composition of intestinal fungi, called the mycobiome, and their association with other diseases. ${ }^{38}$ An increase in mycobiota populations and translocation of fungal $\beta$-glucan into systemic circulation have been recently shown in an animal model of ALD. Moreover, antifungal treatment resulted in an improvement in alcohol-induced liver injuries. This effect was mediated by $\beta$-glucan, a fungal cell wall polysaccharide, which induces liver inflammation via the C-type lectin-like receptor (CLEC7A) on Kupffer cells. ${ }^{39}$

In humans, chronic alcohol consumption is associated with decreased fungal diversity, Candida overgrowth, and the translocation of fungal products, as suggested by an increase in the levels of anti-Saccharomyces cerevisiae IgG antibodies (ASCA). ${ }^{39}$ Moreover, ASCA levels correlated with the mortality of patients with alcoholic cirrhosis in this study. Another study reported fungal dysbiosis associated with cirrhosis and the authors proposed a combined bacterial-fungal dysbiosis metric, the Bacteroidetes/ Ascomycota ratio, which could independently predict 90-day hospitalizations in cirrhotic patients. However, only a small subset of patients in this study had alcoholic cirrhosis and these results are yet to be confirmed in a large cohort of alcoholic cirrhotic patients. ${ }^{40}$

\section{PREBIOTICS, PROBIOTICS, OR FECAL MICRO- BIOTA TRANSFER (FMT) TO TREAT ALD}

Several probiotics have been tested in rodent models and several human trials to slow the progression of ALD. The first use of probiotics in ALD was performed in a rodent alcohol intake model using Lactobacillus $G G$, which improved gut leakiness and liver inflammation. ${ }^{41-43}$ The addition of oat fiber or a supernatant of Lactobacillus $G G$ induced similar results, suggesting that bacterial products were partially involved in the protective mechanisms. ${ }^{21,44,45}$ VSL\#3, a mixture of eight probiotic strains (mainly Lactobacillus and Bifidobacterium), also improved liver lesions in
ALD in humans and rodents. ${ }^{46,47}$ Several other probiotic strains have been tested and also improved liver lesions in rodents. They are well summarized in a recent review. ${ }^{48}$ More recently, A. muciniphila administration prevented hepatic injury, steatosis, and neutrophil infiltration in a mouse model of ALD. These effects were due to an improvement in gut leakiness, enhanced mucus thickness, and increased expression of tight-junction proteins. Furthermore, $A$. muciniphila treatment also improved hepatic injury and neutrophil infiltration in already established ALD. ${ }^{49}$

Variability in the susceptibility to develop liver injury has also been observed in a mouse model of ALD. Sensitivity to alcohol-induced liver lesions was associated with specific dysbiosis and a low level of Bacteroides. Modification of the IM in alcohol-sensitive mice by FMT prevented alcohol-induced liver lesions. Among species associated with the resistance to alcohol, Lactobacillus, Bifidobacteria, and Roseburia were identified as targets of interest. ${ }^{33}$ Recently, a pilot trial that tested FMT in SAH patients ineligible for corticoid therapy showed promising results, suggesting improved survival. However, the small sample size of the study and the use of historical controls limit its interpretation. A phase III trial is ongoing in India (NCT03091010).

Prebiotic use has also been tested and showed that fructo-oligosaccharides (FOS) improved rodent alcohol-induced liver damage in a mouse model of ALD. ${ }^{22}$ The most recent data concern the preventive role of pectin in ALD, in which pectin treatment of alcohol-fed mice restored the level of Bacteroides and completely prevented the development of liver injury. ${ }^{33}$

Altogether, these studies highlight the promise of controlling the IM by pre- or probiotic treatments. However, further studies are needed to understand the mechanisms involved in bacteriarelated gut and liver damage.

\section{IM IN ADVANCED LIVER DISEASE}

The analysis of the IM in fibrosis and cirrhosis during the course of ALD is scarce, as the analysis has been mostly performed independently of the first cause of the disease. Patients with cirrhosis have distinct fecal microbial communities relative to healthy individuals. The authors of a recent study using "shotgun" metagenomics to compare patients with alcoholic cirrhosis to healthy controls found a decrease in the abundance of Parabacteroides, Prevotella, Clostridium, and Bacteroides and an increase in that of LactobacilIus and Bifidobacterium.$^{50}$ Other studies also found a decrease in the abundance of Bacteroidetes in cirrhotic patients (24 HBV and 
12 alcohol), whereas Proteobacteria and Fusobacteria were more abundant. ${ }^{51}$ Among cirrhotic patients, the abundance of Prevote/laceae was greater in alcoholic patients than those infected with HBV. In addition, the prevalence of potentially pathogenic bacteria, such as Enterobacteriaceae and Streptococcaceae, and the reduction of beneficial populations, such as Lachnospiraceae, in patients with cirrhosis, may affect the prognosis. ${ }^{51}$ Moreover, $54 \%$ of the IM bacteria in cirrhosis are of oral origin, suggesting an invasion of the gut from the mouth in liver cirrhosis. ${ }^{52}$

Metabolic pathways have also been studied in cirrhosis by metagenomic profiling. The expression of IM genes involved in gluconeogenesis and lipid metabolism were found to be enriched in patients with cirrhosis, whereas that of genes involved in bile acid metabolism was decreased. ${ }^{53}$ Another study revealed specific clusters representing cognate bacterial species: 28 enriched in cirrhotic patients and 38 in control individuals. Among bacterial genes, those involved in denitrification, nitrate assimilation or dissimilation, GABA biosynthesis, heme biosynthesis, and phosphotransferase systems were associated with liver cirrhosis. ${ }^{52}$ Another study showed gene expression of the IM in ALD patients to be significantly enriched in functions related to alcohol metabolism relative to healthy controls. Most of the genes belonged to the family of alcohol, aldehyde, and acetaldehyde dehydrogenases. ${ }^{50}$

Genetic and functional biomarkers specific for liver cirrhosis have been revealed by a comparison with biomarkers for type 2 diabetes and inflammatory bowel disease. Fifteen biomarkers were specifically associated with cirrhosis. However, generalization of these results is difficult, as the number of patients was low and the cause of cirrhosis was mainly restricted to hepatitis $B$ virus infection. ${ }^{52}$

The survival rate of cirrhotic patients depends on the associated complications, including episodes of hepatic encephalopathy (HE), ascites, or infections, which all appear to be associated with specific bacterial communities. ${ }^{54}$

HE and infectious complications have been shown to be associated with a high level of Enterobacteriaceae. ${ }^{55}$ Alcaligeneceae, bacteria specifically involved in urea metabolism, were also more abundant in HE patients. In a prospective clinical trial, VSL\#3 efficiently prevented $\mathrm{HE}$ in cirrhotic patients, suggesting that the dysbiosis observed in HE may have a causative role. ${ }^{56}$ Conversely, this treatment did not affect portal pressure. ${ }^{57}$ Moreover, subjects in a recent open-label, randomized clinical trial that compared FMT to standard care in recurrent HE showed improved cognition, increased diversity, and a higher abundance of beneficial taxa with FMT, leading to reduced hospitalizations. ${ }^{58}$
Viable bacteria have been found in the ascites of patients with decompensated cirrhosis, including several species of Propionobacterium, Pseudomonas, and Staphylococcus, which normally colonizes the skin. ${ }^{59}$ Thus, access to the peritoneal cavity is not limited to the translocation of bacteria from the gut. However, the quantity of bacteria in the ascites was not sufficient to induce spontaneous bacterial peritonitis, although there was a relationship between the bacterial species and the severity of the disease. $^{59}$

There is also a decrease in overall microbial diversity and richness in acute-on chronic liver failure (ACLF). These patients had a lower abundance of Bacteroidaceae, Ruminococcaceae, and Lanchnospiraceae, but a higher abundance of Pasteurellaceae, Streptococcaceae, and Enterecoccaceae. Moreover, the relative abundance of Pasteurellaceae was an independent predictor of mortality. ${ }^{60}$

A cirrhosis dysbiosis ratio (CDR) has been developed to evaluate the severity of the condition. ${ }^{54}$ Enterobacteriaceae is the most prevalent family modified in the CDR and is specifically associated with the complications of cirrhosis due to the production of potent endotoxins. The level of fecal Bacteroidaceaeae and Clostridiales XIV may predict the risk of hospitalization in patients with cirrhosis, independently of other classical clinical predictors, such as the model for end-stage disease (MELD) score, $H E$, or the intake of proton-pump inhibitors. ${ }^{61}$ However, these studies included patients with cirrhosis of various etiologies. Moreover, a randomized clinical trial using Lactobacillus $G G$ in cirrhotic patients demonstrated that altering the composition of the IM through the use of probiotics improved dysbiosis and endotoxemia. ${ }^{62}$

\section{CONCLUSION}

Recent advances in microbiota research have shown that intestinal bacteria play a crucial role in liver pathology. Changes in the structure, composition, and function of these bacteria are important factors related to ALD. Our understanding of the clinical significance of dysbiosis in ALD is starting to take shape. It will, however, be crucial to consider the possible association between host genetics and/or epigenetic factors that play a potential role in liver injury. Further studies are needed, combining metagenomics, metatranscriptomics, and metabolomics with longitudinal studies, along with standardization of these techniques, to better understand the role of microbiota alterations in the development of ALD. 
Anne-Marie Cassard, et al. Microbiota in alcoholic liver disease

Many studies already suggest that targeting the IM through the use of pro-, pre-, or symbiotics or FMT may be an effective treatment for ALD. There are several ongoing trials in ALD using these strategies: antibiotics (rifaximin, amoxicilline, ciprofloxacine), probiotics (Lactobacillus GG), colostrum, and FMT (clinicaltrials.org). The use of prebiotics, specifically pectin, which can prevent liver injury in rodent models, appears to be promising, as it is a food product that can be easily and safely used in humans.

Larger studies, incorporating liver biopsies, standardized dose administration and duration, and an analysis of the long-term impact on the IM are clearly needed to validate these initial promising results. Indeed, "microbial hepatology" is a new and promising field of tremendous interest to scientists, physicians, and patients that will improve the management of ALD patients.

\section{Authors contribution}

AMC and DC have all contributed to the design and the writing of the review.

\section{Financial support}

INSERM, Univ Paris-Sud/Paris Saclay, SNFGE (Société Nationale Francophone de Gastro-entérologie), AFEF (French Association for the Study of Liver), IREB/FRA (Institut de Recherches sur les Boissons/Fondation pour la recherche en alcoologie), IRIS (Institut de Recherches Internationales Servier), ERAB (The European Foundation for Alcohol Research), FRM (Fondation pour la Recherche Médicale) and Biocodex. INSERM U996 is a member of the Laboratory of Excellence LERMIT supported by a grant from the Agence Nationale de la Recherche (ANR-10-LABX-33).

\section{Acknowledgement}

The authors thank the members of the U996 team and especially Prof Gabriel Perlemuter.

\section{Conflicts of Interest}

The authors have no conflicts to disclose.

\section{REFERENCES}

1. Voican CS, Njiké-Nakseu M, Boujedidi H, Barri-Ova N, BouchetDelbos L, Agostini $\mathrm{H}$, et al. Alcohol withdrawal alleviates adipose tissue inflammation in patients with alcoholic liver disease. Liver Int 2015;35:967-978

2. European Association For The Study Of The Liver. EASL-EORTC clini- cal practice guidelines: management of hepatocellular carcinoma. J Hepatol 2012;56:908-943.

3. Thursz MR, Forrest EH, Ryder S. Prednisolone or Pentoxifylline for Alcoholic Hepatitis. N Engl J Med 2015;373:282-283.

4. Mathurin P, Moreno C, Samuel D, Dumortier J, Salleron J, Durand F, et al. Early liver transplantation for severe alcoholic hepatitis. N Engl J Med 2011;365:1790-1800.

5. Atkinson SR, Way MJ, McQuillin A, Morgan MY, Thursz MR. Homozygosity for rs738409:G in PNPLA3 is associated with increased mortality following an episode of severe alcoholic hepatitis. J Hepatol 2017;67:120-127.

6. Buch S, Stickel F, Trépo E, Way M, Herrmann A, Nischalke HD, et al. A genome-wide association study confirms PNPLA3 and identifies TM6SF2 and MBOAT7 as risk loci for alcohol-related cirrhosis. Nat Genet 2015:47:1443-1448.

7. Stickel F, Moreno C, Hampe J, Morgan MY. The genetics of alcohol dependence and alcohol-related liver disease. J Hepatol. 2017;66:195-211.

8. Adachi Y, Moore LE, Bradford BU, Gao W, Thurman RG. Antibiotics prevent liver injury in rats following long-term exposure to ethanol. Gastroenterology 1995;108:218-224.

9. Bode C, Kugler V, Bode JC. Endotoxemia in patients with alcoholic and non-alcoholic cirrhosis and in subjects with no evidence of chronic liver disease following acute alcohol excess. J Hepatol 1987:4:8-14.

10. Mathurin P, Deng QG, Keshavarzian A, Choudhary S, Holmes EW Tsukamoto $\mathrm{H}$. Exacerbation of alcoholic liver injury by enteral endotoxin in rats. Hepatology 2000;32:1008-1017.

11. Nanji AA, Khettry U, Sadrzadeh SM, Yamanaka T. Severity of liver injury in experimental alcoholic liver disease. Correlation with plasma endotoxin, prostaglandin E2, leukotriene B4, and thromboxane B2. Am J Pathol 1993;142:367-373.

12. Hritz I, Mandrekar P, Velayudham A, Catalano D, Dolganiuc A, Kodys $K$, et al. The critical role of toll-like receptor (TLR) 4 in alcoholic liver disease is independent of the common TLR adapter MyD88. Hepatology 2008;48:1224-1231.

13. Keshavarzian A, Fields JZ, Vaeth J, Holmes EW. The differing effects of acute and chronic alcohol on gastric and intestinal permeability. Am J Gastroenterol 1994;89:2205-2211.

14. Parlesak A, Schäfer C, Schütz T, Bode JC, Bode C. Increased intestinal permeability to macromolecules and endotoxemia in patients with chronic alcohol abuse in different stages of alcohol-induced liver disease. J Hepatol 2000;32:742-747.

15. Rao R. Endotoxemia and gut barrier dysfunction in alcoholic liver disease. Hepatology 2009;50:638-644.

16. Elamin EE, Masclee AA, Dekker J, Jonkers DM. Ethanol metabolism and its effects on the intestinal epithelial barrier. Nutr Rev 2013;71:483-499. 
17. Grewal RK, Mahmood A. Ethanol induced changes in glycosylation of mucins in rat intestine. Ann Gastroenterol 2009;22:178-183.

18. Ferrier L, Bérard F, Debrauwer L, Chabo C, Langella $P$, Buéno $L$, et al. Impairment of the intestinal barrier by ethanol involves enteric microflora and mast cell activation in rodents. Am J Pathol 2006;168:1148-1154.

19. Ulluwishewa D, Anderson RC, McNabb WC, Moughan PJ, Wells JM, Roy NC. Regulation of tight junction permeability by intestinal bacteria and dietary components. J Nutr 2011;141:769-776.

20. Bull-Otterson L, Feng W, Kirpich I, Wang Y, Qin X, Liu Y, et al. Metagenomic analyses of alcohol induced pathogenic alterations in the intestinal microbiome and the effect of Lactobacillus rhamnosus GG treatment. PLoS One 2013;8:e53028.

21. Mutlu E, Keshavarzian A, Engen P, Forsyth CB, Sikaroodi M, Gillevet P. Intestinal dysbiosis: a possible mechanism of alcohol-induced endotoxemia and alcoholic steatohepatitis in rats. Alcohol Clin Exp Res 2009;33:1836-1846.

22. Yan AW, Fouts DE, Brandl J, Starkel P, Torralba M, Schott E, et al. Enteric dysbiosis associated with a mouse model of alcoholic liver disease. Hepatology 2011;53:96-105.

23. Mutlu EA, Gillevet PM, Rangwala $H$, Sikaroodi M, Naqvi A, Engen $\mathrm{PA}$, et al. Colonic microbiome is altered in alcoholism. Am J Physiol Gastrointest Liver Physiol 2012;302:G966-G978.

24. Llopis M, Cassard AM, Wrzosek L, Boschat L, Bruneau A, Ferrere G, et al. Intestinal microbiota contributes to individual susceptibility to alcoholic liver disease. Gut 2016;65:830-839.

25. Dorrestein PC, Mazmanian SK, Knight R. Finding the missing links among metabolites, microbes, and the host. Immunity 2014;40:824-832.

26. Chen P, Torralba M, Tan J, Embree M, Zengler $K$, Stärkel $P$, et al. Supplementation of saturated long-chain fatty acids maintains intestinal eubiosis and reduces ethanol-induced liver injury in mice. Gastroenterology 2015;148:203-214. e216.

27. Kirpich IA, Petrosino J, Ajami N, Feng W, Wang Y, Liu Y, et al. Saturated and Unsaturated Dietary Fats Differentially Modulate EthanolInduced Changes in Gut Microbiome and Metabolome in a Mouse Model of Alcoholic Liver Disease. Am J Pathol 2016;186:765-776.

28. Hylemon PB, Zhou H, Pandak WM, Ren S, Gil G, Dent P. Bile acids as regulatory molecules. J Lipid Res 2009;50:1509-1520.

29. Couch RD, Dailey A, Zaidi F, Navarro K, Forsyth CB, Mutlu E, et al. Alcohol induced alterations to the human fecal VOC metabolome. PLoS One 2015;10:e0119362.

30. Fouts DE, Torralba M, Nelson KE, Brenner DA, Schnabl B. Bacterial translocation and changes in the intestinal microbiome in mouse models of liver disease. J Hepatol 2012;56:1283-1292.

31. Hartmann P, Chen P, Wang HJ, Wang L, McCole DF, Brandl K, et al. Deficiency of intestinal mucin-2 ameliorates experimental alcoholic liver disease in mice. Hepatology 2013;58:108-119.

32. Wang L, Fouts DE, Stärkel P, Hartmann P, Chen P, Llorente C, et al.
Intestinal REG3 Lectins Protect against Alcoholic Steatohepatitis by Reducing Mucosa-Associated Microbiota and Preventing Bacterial Translocation. Cell Host Microbe 2016;19:227-239.

33. Ferrere G, Wrzosek L, Cailleux F, Turpin W, Puchois V, Spatz M, et al. Fecal microbiota manipulation prevents dysbiosis and alcoholinduced liver injury in mice. J Hepatol 2017;66:806-815.

34. Little TJ, Feinle-Bisset C. Effects of dietary fat on appetite and energy intake in health and obesity--oral and gastrointestinal sensory contributions. Physiol Behav 2011;104:613-620.

35. Mithieux G. Crosstalk between gastrointestinal neurons and the brain in the control of food intake. Best Pract Res Clin Endocrinol Metab 2014;28:739-744.

36. Bauer KC, Huus KE, Finlay BB. Microbes and the mind: emerging hallmarks of the gut microbiota-brain axis. Cell Microbiol. 2016;18:632-644.

37. Leclercq S, Matamoros S, Cani PD, Neyrinck AM, Jamar F, Stärkel P, et al. Intestinal permeability, gut-bacterial dysbiosis, and behavioral markers of alcohol-dependence severity. Proc Natl Acad Sci U S A 2014;111:E4485-4493.

38. Wang ZK, Yang YS, Stefka AT, Sun G, Peng LH. Review article: fungal microbiota and digestive diseases. Aliment Pharmacol Ther 2014;39:751-766.

39. Yang AM, Inamine T, Hochrath K, Chen P, Wang L, Llorente $C$, et al. Intestinal fungi contribute to development of alcoholic liver disease. J Clin Invest 2017;127:2829-2841.

40. Bajaj JS, Liu EJ, Kheradman R, Fagan A, Heuman DM, White M, et al. Fungal dysbiosis in cirrhosis. Gut 2017 Jun 3. [Epub ahead of print]

41. Forsyth CB, Farhadi A, Jakate SM, Tang Y, Shaikh M, Keshavarzian A. Lactobacillus GG treatment ameliorates alcohol-induced intestinal oxidative stress, gut leakiness, and liver injury in a rat model of alcoholic steatohepatitis. Alcohol 2009;43:163-172.

42. Nanji AA, Khettry U, Sadrzadeh SM. Lactobacillus feeding reduces endotoxemia and severity of experimental alcoholic liver (disease). Proc Soc Exp Biol Med 1994;205:243-247.

43. Keshavarzian A, Choudhary S, Holmes EW, Yong S, Banan A, Jakate $S$, et al. Preventing gut leakiness by oats supplementation ameliorates alcohol-induced liver damage in rats. J Pharmacol Exp Ther 2001;299:442-448.

44. Wang Y, Kirpich I, Liu Y, Ma Z, Barve S, McClain CJ, et al. Lactobacillus rhamnosus $\mathrm{GG}$ treatment potentiates intestinal hypoxia-inducible factor, promotes intestinal integrity and ameliorates alcohol-induced liver injury. Am J Pathol 2011;179:2866-2875.

45. Wang Y, Liu Y, Kirpich I, Ma Z, Wang C, Zhang M, et al. Lactobacillus rhamnosus $G G$ reduces hepatic TNFalpha production and inflammation in chronic alcohol-induced liver injury. J Nutr Biochem 2013;24:1609-1615.

46. Chang B, Sang L, Wang Y, Tong J, Zhang D, Wang B. The protective 
effect of VSL\#3 on intestinal permeability in a rat model of alcoholic intestinal injury. BMC Gastroenterol 2013;13:151.

47. Loguercio C, Federico A, Tuccillo C, Terracciano F, D'Auria MV, De Simone $C$, et al. Beneficial effects of a probiotic VSL\#3 on parameters of liver dysfunction in chronic liver diseases. J Clin Gastroenterol 2005;39:540-543.

48. Zhong W, Zhou Z. Alterations of the gut microbiome and metabolome in alcoholic liver disease. World J Gastrointest Pathophysiol 2014;5:514-522.

49. Grander C, Adolph TE, Wieser V, Lowe P, Wrzosek L, Gyongyosi B, et al. Recovery of ethanol-induced Akkermansia muciniphila depletion ameliorates alcoholic liver disease. Gut 2017 May 26. [Epub ahead of print]

50. Dubinkina VB, Tyakht AV, Odintsova VY, Yarygin KS, Kovarsky BA, Pavlenko $A V$, et al. Links of gut microbiota composition with alcohol dependence syndrome and alcoholic liver disease. Microbiome 2017;5:141.

51. Chen Y, Yang F, Lu H, Wang B, Chen Y, Lei D, et al. Characterization of fecal microbial communities in patients with liver cirrhosis. Hepatology 2011;54:562-572.

52. Qin N, Yang F, Li A, Prifti E, Chen Y, Shao L, et al. Alterations of the human gut microbiome in liver cirrhosis. Nature 2014;513:59-64.

53. Wei X, Yan X, Zou D, Yang Z, Wang X, Liu W, et al. Abnormal fecal microbiota community and functions in patients with hepatitis $B$ liver cirrhosis as revealed by a metagenomic approach. BMC Gastroenterol 2013;13:175

54. Bajaj JS, Heuman DM, Hylemon PB, Sanyal AJ, White MB, Monteith $P$, et al. Altered profile of human gut microbiome is associated with cirrhosis and its complications. J Hepatol 2014;60:940-947.
55. Bajaj JS, Ridlon JM, Hylemon PB, Thacker LR, Heuman DM, Smith S, et al. Linkage of gut microbiome with cognition in hepatic encephalopathy. Am J Physiol Gastrointest Liver Physiol 2012;302:G168-G175.

56. Lunia MK, Sharma BC, Sharma P, Sachdeva S, Srivastava S. Probiotics prevent hepatic encephalopathy in patients with cirrhosis: a randomized controlled trial. Clin Gastroenterol Hepatol 2014;12:10031008. e1001.

57. Jayakumar S, Carbonneau M, Hotte N, Befus AD, St Laurent C, Owen R, et al. VSL\#3 (R) probiotic therapy does not reduce portal pressures in patients with decompensated cirrhosis. Liver Int 2013;33:14701477.

58. Bajaj JS, Kassam Z, Fagan A, Gavis EA, Liu E, Cox IJ, et al. Fecal microbiota transplant from a rational stool donor improves hepatic encephalopathy: A randomized clinical trial. Hepatology 2017;66:17271738.

59. Rogers GB, van der Gast CJ, Bruce KD, Marsh P, Collins JE, Sutton J, et al. Ascitic microbiota composition is correlated with clinical severity in cirrhosis with portal hypertension. PLoS One 2013;8:e74884.

60. Chen Y, Guo J, Qian G, Fang D, Shi D, Guo L, et al. Gut dysbiosis in acute-on-chronic liver failure and its predictive value for mortality. J Gastroenterol Hepatol 2015;30:1429-1437.

61. Bajaj JS, Betrapally NS, Hylemon PB, Thacker LR, Daita K, Kang DJ, et al. Gut Microbiota Alterations can predict Hospitalizations in Cirrhosis Independent of Diabetes Mellitus. Sci Rep 2015;5:18559.

62. Bajaj JS, Heuman DM, Hylemon PB, Sanyal AJ, Puri P, Sterling RK, et al. Randomised clinical trial: Lactobacillus GG modulates gut microbiome, metabolome and endotoxemia in patients with cirrhosis. Aliment Pharmacol Ther 2014;39:1113-1125. 